\title{
A hybrid KNN-MLP algorithm to diagnose bipolar disorder
}

\author{
Mozhgan Mohammad Ghasemi ${ }^{1 *}$, Mehdi Khalili ${ }^{2}$ \\ ${ }^{1}$ M.S. Student, Department of Computer and Informatics Payame Noor University \\ ${ }^{2}$ Assistant Professor, Dept. of Computer and Informatics Payame Noor University \\ *Corresponding author E-mail: Mozhgan_7327@yahoo.com
}

Copyright () 2015 Mozhgan Mohammad Ghasemi , Mehdi Khalili. This is an open access article distributed under the Creative Commons Attribution License, which permits unrestricted use, distribution, and reproduction in any medium, provided the original work is properly cited.

\begin{abstract}
In this paper an attempt has been made to the other corner of the power of neural networks. According to the neural network in the diagnosis of diseases, we use neural network models for diagnosing bipolar disorder; bipolar disorder is the common disorder of depression mood. We have used two neural network models: MLP \& KNN. With different percentages of the implementation of neural network models is discussed. And the error was calculated for each model. We can by using the MLP model achieve an error of $16 \%$ for the diagnosis of bipolar disorder.
\end{abstract}

Keywords: Bipolar Disorder; MLP; KNN.

\section{Introduction}

Artificial neural networks are finding many uses in the medical diagnosis application [1].To streamline the diagnostic process in daily routine and avoid misdiagnosis, artificial intelligence Methods (especially computer aided diagnosis and artificial neural networks) can be employed. These adaptive learning algorithms can handle diverse types of medical data and integrate them into categorized outputs [2]. In 2004, the global burden of disease report (GBD) revealed, psychological disorders accounted for $14 \%$ of the total global disease burden [3]. Depression is the most prevalent mental health disorder affecting approximately 100 million people globally [4]. It has become a general disease in the world due to the promotion of life quality and technology development. Most of people are not aware of the possibility of getting depressed himself in daily life. To accurately diagnose getting depressed becomes an important issue [5]. "Depression" is a psychological disorder in which the patient's activities severely reduced and in fact, he was not motivated to do many things. Depressed person's energy and life skills he will fall, and his concentration is greatly reduced. However, sometimes aggressive, sometimes it is frustrating. His guilt is very strong. In addition, the patient will return your goals in life and reduce social activities and production. [6]. The analysis of scientific investigations shows that very limited works have been reported on diagnosis of depression using neural networks structure, for example :In [7], ten different types of classification algorithms are applied to depression diagnosis and their performance is compared, through a set of experiments on SMRI brain scans. In the experiments, a procedure is developed to measure the performance of these algorithms and an evaluation method is employed to evaluate and compare the performance of the classifiers. In [8], a machine learning method is proposed for automatically finding psychiatric diagnostic rules. It is proposed that a genetic algorithm (GA) system can find symbolic, easily readable rules that could be used by psychiatric clinicians. Diagnosis of major depressive disorder is considered. A sample of 320 subjects with symptom information and pre-assigned diagnosis is used to train a GA model and two other statistical models, discriminant analysis and logistic regression. Each model is able correctly to classify more than $91 \%$ of cases. The GA model performs best of the three methods and yields readable, non-numeric rules. In [9], presents psyche system, a personal, cost-effective, multi-parametric monitoring system based on textile platforms and portable sensing devices for the long term and short term acquisition of data from selected class of patients affected by mood disorders. The acquired data will be processed and analyzed in the established platform that takes into account the Electronic Health Records (EHR) of the patient, a personalized data referee system, as well as medical analysis in order to verify the diagnosis and help in prognosis of the illness. In [10], the authors conducted a resting-state functional connectivity 
fMRI study of 35 bipolar disorder and 25 schizophrenia patients, to investigate the relationship between bipolar disorder and schizophrenia, using computation of the mean connectivity within and between five neural networks: default mode (DM), fronto-parietal (FP), cingulo-opercular (CO), cerebellar (CER), and salience (SAL). They found that across groups, connectivity was decreased between CO-CER, to a larger degree in schizophrenia than in bipolar disorder. Also, in schizophrenia, there was also decreased connectivity in CO-SAL, FP-CO, and FP-CER, while bipolar disorder showed decreased CER-SAL connectivity. Disorganization symptoms were predicted by connectivity between COCER and CER-SAL.

In this paper, the use of neural network models (MLP, KNN), discussed for diagnosis of bipolar disorder. First, the parameters needed to diagnose detected. The data used in this study for education are also used for network testing. After the design of neural network models and test the network, according to error of models for diagnose, we choose the best model for this disorder, it is obvious, best model had the minimum error than other models.

\section{Definition idioms}

In this section, all terms been used in this article, we have introduced:

\subsection{Neural network}

The main idea of machine learning WarenMcCulloch and Walter Pitts in 1940 by taking the model of the human brain neurons were raised. [11]. Set the input weights of each neuron makes learning neural network is an artificial neural network can be a single layer or multiple layers, resistance and damage tolerance can be learned, being (i.e., the ability to adjust the weight LAN), generalize, because of the high speed parallel processing, coping with change. Feature of Neural network system and non-linear system model. This article is based on a neural network can be used as an efficient tool in identifying psychiatric patterns. [12][13].

\subsection{MLP model}

A multilayer perceptron (MLP) is a feed forward artificial neural network model that maps sets of input data onto a set of appropriate outputs [14]. This type of network is trained with the back propagation learning algorithm. MLPs are widely used for pattern classification, recognition, prediction and approximation. Multi-Layer Perceptron can solve problems which are not linearly separable [15].

\subsection{KNN model}

The k-Nearest-Neighbors (KNN) is a simple but effective method for classification. The major drawbacks with respect to KNN are (1) its low efficiency - being a lazy learning method prohibits it in many applications such as dynamic web mining for a large repository, and (2) its dependency on the selection of a "good value" for k [16].

\subsection{Depression}

Depression is a most common severe mental disturbance health disorder causing high societal costs. In clinical practice rating for depression depends almost on self-questionnaires and clinical patient history report opinion [17]. Depression is a feature of some psychiatric syndromes such as bipolar disorder but it may also be a normal reaction to life events such as bereavement, a symptom of some bodily ailments or a side effect of some drugs and medical treatments [18].

\subsection{Bipolar disorder}

Bipolar disorder (i.e., bipolar disorder I, bipolar disorder II, temperamental cyclical disorder and bipolar disorder which is not otherwise identified) requires mania history courses, hybrid courses, courses that usually accompanies Hypo mania despite (or history) major depressive episodes [19]. Bipolar Disorder Type I: according to one or more courses or mixed Mania usually associated with major depressive episodes are characterized. Bipolar disorder II: according to one or more periods of major depression associated with at least one course Hypo mania be determined. Periodic disturbance: According to multiple periods Hypo mania symptoms for at least 2 years Mania not match the criteria of diagnosis and the presence of numerous periods with depressive symptoms that do not meet the criteria for a diagnosis of major depression, is by characterized [20] [21]. 


\section{Proposed algorithm}

At this stage, the parameters obtained from the records of patients as well as related articles [22], [23]; Simulation of neural network models has been conducted. The parameters used in this paper can be pointed to "depression mood", "feelings of guilt", "risky driving", "risky sex", "Jump thoughts", "lack of sleep”, "high joy”, work disorder", “cry”, "lack of success", "sleepless", "sad", "poor judgment", "lack of focus", etc. In this article use the 47 parameters for diagnosis of bipolar disorder. The data used in this study for education are also used for network testing. Then, depending on the model selected (MLP or KNN), the simulation is performed. After simulation, Error for each of the models calculated with respect to different percentages training. Because networks training with of different levels, we can calculate the least error and get the best of education. And, in the end, select the best model for diagnose of bipolar disorder. Following the results of simulations of the two models is shown.

\subsection{MLP model results}

To implement this model using, 10 input neurons, 5 neurons in the middle and 4 output neurons. In the fact, 3 output neurons show the 3 class for diagnose the bipolar disorder. These classes are BD (I), BD (II) and health. This model simulation with the different percentage training. The results of this model show in "Table.1". And for the best percentage training, performance graph show in "figure.1".

As can be seen in "Table.1", for 70 percentage training has the minimum error and it is the best error with this model for diagnosis bipolar disorder, also for 60 percentage training has the maximum error and it not suitable.

Table 1: Error of MLP Model

\begin{tabular}{ll}
\hline Network error & Percentage training \\
\hline 0.19 & 40 \\
0.19 & 50 \\
0.22 & 60 \\
0.16 & 70 \\
0.17 & 80 \\
0.19 & 90 \\
\hline
\end{tabular}

"Figure.1" shows for 9 epoch training MLP model in seventh epoch has the best validation. Validation of this epoch is 0.049 .

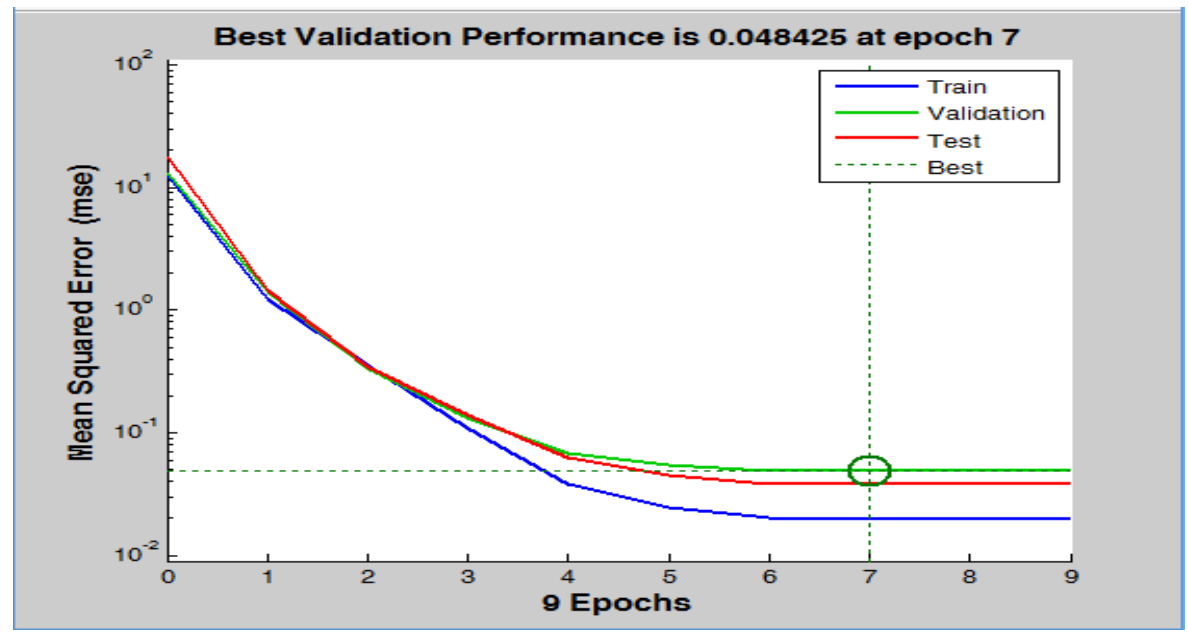

Fig. 1: Performance Graph for MLP Model

\subsection{KNN model results}

To implement this model using, 3 parameters for training, testing and grouping the data. This model has the 3 class output. These classes are BD (I), BD (II), and healthy. This model simulation with the different percentage training. The results of this model show in "Table.2". And for the best percentage training, performance graph show in "figure.2". As can be seen in "Table.2", for 70 percentage training have the minimum error and it is the best error with this model for diagnosis bipolar disorder, also for 90 and 50 percentage training have the maximum error and it not suitable. 
Table 2: Error of KNN Model

\begin{tabular}{ll}
\hline Network error & Percentage training \\
\hline 0.24 & 40 \\
0.25 & 50 \\
0.24 & 60 \\
0.21 & 70 \\
0.23 & 80 \\
0.25 & 90 \\
\hline
\end{tabular}

"Figure.2" shows for 8 epoch training KNN model in fourth epoch has the best validation. Validation of this epoch is 0.050 .

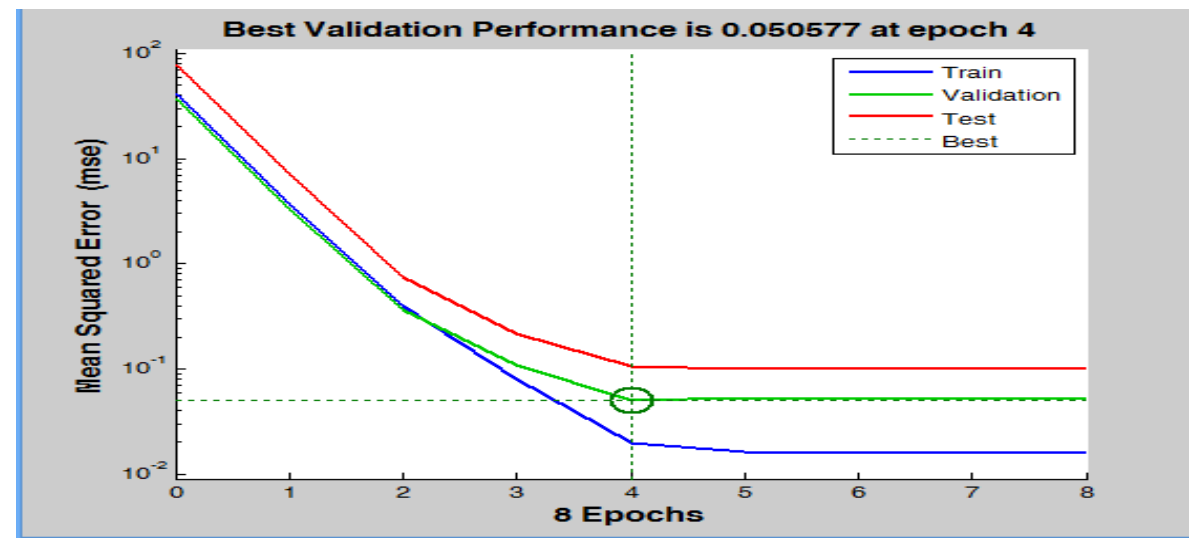

Fig. 2: Performance Graph for KNN Model

\subsection{Compare the models}

In "Figure.3" have the compare MLP \& KNN models. It can be seen for both models in $70 \%$ training have the minimum error and it the best percentage training. But, the total compares in "Table.3" shows. In this table obtained with MLP model in diagnose with best percentage training have the $16 \%$ error and with using the KNN model have the $21 \%$ error. Thus, the MLP model has the best diagnose for bipolar disorder.

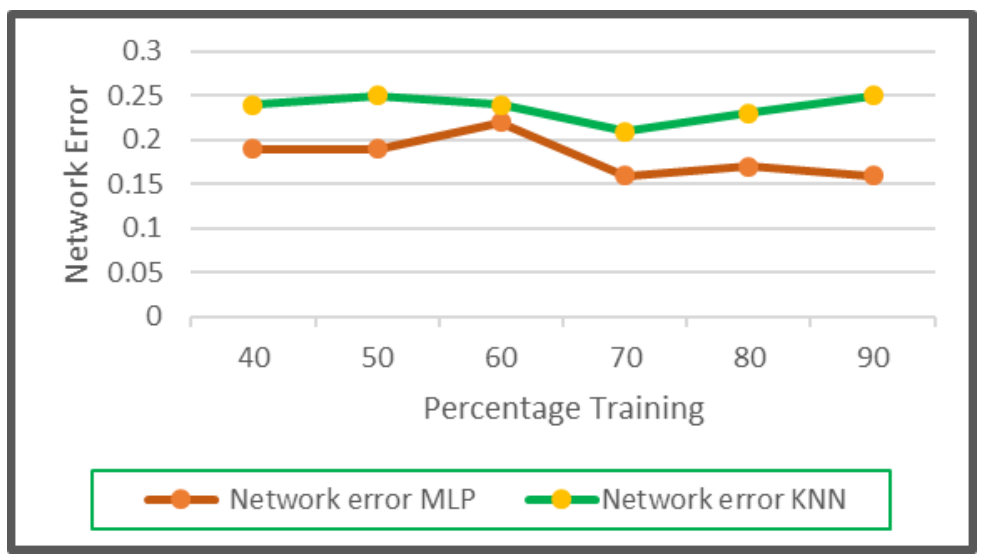

Fig. 3: Compare MLP \& KNN Error

Table 3: Compare Models

\begin{tabular}{ll}
\hline Percentage Error & Model \\
\hline $16 \%$ & MLP \\
$21 \%$ & KNN \\
\hline
\end{tabular}

\section{Conclusion}

In this paper an attempt is made to use the parameters required for the diagnosis of bipolar disorder, deal to implementation of neural network models. The models used in this paper are KNN \& MLP. With the available data to train and test the models discussed. Error for each models obtained with different percentage education. And saw with $70 \%$ training have the minimum error for testing network. Also, with using the MLP model for diagnose have the $16 \%$ 
error and with using the KNN model have the $21 \%$ error. And we can conclude by using the MLP model can we have better diagnosis for bipolar disorder.

\section{References}

[1] Fukushima, K., "Neocognitron: A self-organizing neural network model for a mechanism of pattern recognition unaffected by shift in position". Biological Cybernetics, vol. 36 (4), 2014, pp. 93

[2] American.Psychiatric.Association, "Diagnostic and statistical manual of mental disorder", 4th edition, American. Psychiatric. Association, 2000 .

[3] Yun Lin, Yuan Zhang. "Prediction of number of depression patients based on neural network". Software Engineering and Service Science (ICSESS). DOI. 10.1109/ICSESS.2012.6269538.pp. 599-602.

[4] S.Chattopadhyay, P.Kaur, F.Rabhi, U, Rajendra Acharya."An Automated System to Diagnose the Severity of Adult Depression". Emerging Application of Information Technology (EAIT). DOI. 10.1109/EAIT.2011.17.2011. pp. 121-124.

[5] Yue-Shan.Chang, Dept. of Comput. Sci. \& Inf. Eng., Wan-Chun Hung, Tong-Ying Juang, "Depression Diagnosis Based on Ontologies and Bayesian Networks. Systems Man and Cybernetics (SMC)". IEEE International Conference. DOI. 10.1109/SMC.2013.589.2013. pp. 34523457.

[6] G. O. Young, Synthetic structure of industrial plastics, New York: McGraw-Hill, 1964

[7] Kipli, K., Sch. of Eng. Sch. of Inf. Technol., Deakin Univ., Waurn Ponds, VIC, Australia, Kouzani, A.Z., Yong Xiang, “An Empirical Comparison of Classification Algorithms for Diagnosis of Depression from Brain SMRI Scans",Advanced Computer Science Applications and Technologies (ACSAT), 2013 International Conference on, DOI. 10.1109/ACSAT.2013.72, 2013, PP. 333-336.

[8] Chapman, C.N., Dept. of Psychol., Tulsa Univ., Deaton, L. Harris, A., Robinson, N., " A genetic algorithm system to find symbolic rules for diagnosis of depression", Evolutionary Computation, 1999. CEC 99. Proceedings of the 1999 Congress on, VOL. 1, DOI. 10.1109/CEC.1999.781917, 1999, PP. 145-149.

[9] Paradiso, R., Bianchi, A.M., Lau, K., Scilingo, E.P."PSYCHE: Personalised monitoring systems for care in mental health", Engineering in Medicine and Biology Society (EMBC), Annual International Conference of the IEEE, DOI. 10.1109/IEMBS.2010.5627469, 2010, PP. 182193.

[10] Mamah, D; Barch, DM.”Resting state functional connectivity of five neural networks in bipolar disorder and schizophrenia". J Affect Disord; DOI. 10.1016/j.jad.2013.01.051. 2013. Pp.214-223. http://dx.doi.org/10.1016/j.jad.2013.01.051.

[11] phil.picton, "introduction to neura network", macmilan publishers limited, 1994, pp.145-150.

[12] Filippo.Amato, Alberto.López, etc. "Artificial Neural Networks in Medical Diagnosis".Vol.11,Journal of Applied Biomedicine, 2013,pp. 4758.

[13] Proceedings 59th ISI World Statistics Congress, 25-30 August 2013, pp.549.

[14] phil.picton, "introduction to neural network", Macmillan publishers limited, 1994

[15] Andreoli de marchi.R. "A multilayer perceptron controller applied to the control of a doubly fed induction generator. Sustianable Energy". IEEE transaction on. DOI. 10.1109/TSTE.2013.2293621; VOL. 2.2013. pp. 1949-3029

[16] Gongde Guo, Hui Wang. "KNN model-based approach in classification", OTM Confederated International Conferences, CoopIS, DOA, and ODBASE 2003, Catania, Sicily, Italy, November 3-7, 2003. Proceedings, 2003, DOI. 10.1007/978-3-540-39964-3_62, pp. 986-996. http://dx.doi.org/10.1007/978-3-540-39964-3 62.

[17] Mantri,S. "clinical depression analysis using speech features", Emerging Trends in Engineering and Technology (ICETET), 2013 6th International Conference on, DOI. 10.1109/ICETET.2013.32, 2013, pp. 111-112.

[18] G. Valenza, C. Gentili, A. Lanatà, and E. P. Scilingo, "Mood recognition in bipolar patients through the PSYCHE platform: Preliminary evaluations and perspectives," Artif. Intell. Med., vol. 57, no. 1, pp. 49-58, 2013. http://dx.doi.org/10.1016/j.artmed.2012.12.001

[19] timothy.J,E.Jerry.Phares,etc. "clinical psychology concepts,methods,\& profession", 6thed, belmont. calif.wadsworth/ thomas learning, 2001, pp.280-334.

[20] Pfennig.A, Bschor.T, Baghai.T, etc. "S3 guidelines ondiagnostics and therapy of bipolar disorders: developmentprocess andessential recommendations", Vol.83 (5), Nervenarzt, 2012, pp.568-586.

[21] Mana S, Martinot MLP, Martinot JL," Brain Imaging Findings in Children and Adolescents with Mental Disorders", a Cross-sectional Review J Eurpsy, VOL. 25, 2013, PP. 345-354.

[22] Parry, P.I.; Levin, E.C., "Pediatric Bipolar Disorder in an Era of "Mindless Psychiatry"". Journal of Trauma \& Dissociation, VOL. 13 (1), 2013, PP. 51-68. 